Chapter 23

\title{
Quantization as Selection Rather than Eigenvalue Problem
}

\author{
Peter Enders
}

Additional information is available at the end of the chapter

http://dx.doi.org/10.5772/53847

\section{Introduction}

The experimental, in particular, spectroscopic results about atoms brought Bohr [3] to the following "principal assumptions" for a theory of atoms.

1. That the dynamical equilibrium of the systems in the stationary states can be discussed by help of the ordinary mechanics, while the passing of the systems between different stationary states cannot be treated on that basis.

2. That the latter process is followed by the emission of a homogeneous radiation, for which the relation between the frequency and the amount of energy emitted is the one given by Planck's theory." (p. 7)

Assumption (1) states a strange contraposition of the conservation and of the changes of stationary states. Indeed, the conservation of energy - a generalization of Newton's Law 1 holds true quite general, while the change of stationary states along Newton's Law 2 is restricted to classical mechanics $(\mathrm{CM})$. A smooth transition from representations of $\mathrm{CM}$, which axiomatically fix not only the conditions of conservation, but also the manner of change of stationary states and the equation(s) of motion, respectively (Newton, Lagrange, Hamilton), is impossible, as observed also by Heisenberg [30] and Schrödinger [43]. However, in Leonhard Euler's representation of CM [24][25], only the conditions of conservation of stationary states are fixed, while their change has to be described according to the situation under consideration. This makes it suitable to serve as starting point for a smooth transition from classical to quantum mechanics (QM). Euler's principles of the change of stationary states of bodies will be generalized to classical conservative systems as well as to quantum systems. The latter will be used for deriving the time-dependent from the time-independent Schrödinger equation. 
In his pioneering papers 'Quantization as Eigenvalue Problem' [43], Schrödinger has posed four requirements.

1. The "quantum equation" should "carry the quantum conditions in itself" (Second Commun., p. 511);

2. There should be a special mathematical method for solving the stationary Schrödinger equation, which accounts for the non-classical character of the quantization problem, ie, which is different from the classical eigenvalue methods for calculating the (eigen)modes of strings, resonators and so on (ibid., p. 513);

3. The derivation should uniquely decide, that the energy rather than the frequency values are discretized (ibid., pp. 511, 519), since only the former means true quantization, while the latter corresponds to the classical discretization mentioned in requirement 2;

4. The use of the classical expressions for the kinetic and potential energies should be justified (Fourth Commun., p. 113).

Schrödinger's requirements 1 and 2 mean, that - contrary to the very title of the papers quantization is actually not an eigenvalue problem. For in the latter the discretization is imposed not by the differential equation itself, but by the boundary conditions, and this is the classical discretization for standing waves in organ pipes etc. I will fulfill all four requirements by treating quantization as a selection problem. The number of stationary states of a quantum system is smaller than that of a classical system [6]. I will describe, (i), the selection of quantum systems out of the set of all mechanical systems and, (ii), the selection of their stationary states out of the continuum of classical stationary states. Earlier arguing [19][10] is improved and extended in several essential points.

\section{Elements of an Eulerian representation of classical mechanics}

\subsection{Euler's axioms}

Leonhard Euler [20-25] was the first to apply the calculus to all areas of mathematics and mechanics of his time, and he developed novel methods, such as the calculus of variations and topology. Moreover, he worked out an axiomatic of mechanics, where only Newton's Law (axiom) 1 concerning the conservation of stationary states is retained as an axiom, while Newton's Laws (axioms) 2 and 3 concerning the change of stationary states are treated as problems to be solved (for a detailed account, see [19][10][45]). This allows for introducing alternative equations of motion without loosing the contact to CM.

The existence of stationary states is postulated in the following axioms (as in Newton's axioms, rotatory motion is not considered).

Axiom E0: Every body is either resting or moving.

This means, that the subsequent axioms E1 and E2 are not independent of each other; they exclude each other and, at once, they are in harmony with each other [22]. 
Axiom E1: A body preserves its stationary state at rest, unless an external cause sets it in motion. Axiom E2: A body preserves its stationary state of straight uniform motion, unless an external cause changes this state.

The stationary-state variable is the velocity vector, $\mathbf{v}$ (the mass of a body is always constant). The equation of stationary state reads $\mathbf{v}=\mathbf{0}$ for the state at rest and $\mathbf{v}=$ const for the state of straight uniform motion. The position, $\mathbf{r}$, is variable of the state of motion, but not of stationary states, because it changes during straight uniform motion, $i e$, in the absence of (external) causes for changing the stationary state [50].

\subsection{Eulerian principles of change of state for single bodies}

Following [21], the changes of position, $\mathbf{r}$, and velocity, $\mathbf{v}$, of a body of mass $m$ subject to the (external) force, $\mathbf{F}$, during the time interval $d t$ are

$$
d\left(\begin{array}{l}
\mathbf{r} \\
\mathbf{v}
\end{array}\right)=\left(\begin{array}{c}
\mathbf{v} d t \\
\frac{1}{m} \mathbf{F} d t
\end{array}\right)=\left(\begin{array}{ll}
\mathbf{0} & \mathbf{1} \\
\mathbf{0} & \mathbf{0}
\end{array}\right)\left(\begin{array}{l}
\mathbf{r} \\
\mathbf{v}
\end{array}\right) d t+\left(\begin{array}{cc}
\mathbf{0} & \mathbf{0} \\
\mathbf{0} & \frac{1}{m} \mathbf{1}
\end{array}\right)\left(\begin{array}{l}
\mathbf{0} \\
\mathbf{F}
\end{array}\right) d t \equiv \mathbf{U}_{i n t}\left(\begin{array}{l}
\mathbf{r} \\
\mathbf{v}
\end{array}\right)+\mathbf{U}_{e x t}\left(\begin{array}{l}
\mathbf{0} \\
\mathbf{F}
\end{array}\right)
$$

The internal transformation, $\mathbf{U}_{\text {int }}$, describes the internal change, $d \mathbf{r}=\mathbf{v} d t$, that is independent of the external force. The external transformation, $\mathbf{U}_{e x t}$ describes the external change, $d \mathbf{v}=(\mathbf{F} / m) d t$, that depends on the external force. These matrices do not commute: $\mathbf{U}_{\text {int }} \mathbf{U}_{\text {ext }} \neq \mathbf{U}_{\text {ext }} \mathbf{U}_{\text {int }}$. This means, that the internal and external transformations are not reducible onto another; the internal and external changes are independent each of another.

Thus, up to order $d t$,

CB1) The changes of stationary-state quantities $(d \mathbf{v})$ are external changes; they explicitly depend solely on external causes (F), but not on state-of-motion quantities (r);

CB2) The change of the stationary-state quantities $(d \mathbf{v})$ do not explicitly depend on the stationary-state quantities (v) themselves;

CB3) The change of state-of-motion quantities $(d \mathbf{r})$ are internal changes; they explicitly depend solely on stationary-state quantities (v) ;

CB4) The change of stationary-state $(d \mathbf{v})$ and of state-of-motion quantities $(d \mathbf{r})$ are independent each of another;

CB5) As soon as the external causes (F) vanish, the body remains in the stationary state assumed at this moment: $Z(t)=$ const $=Z\left(t_{1}\right)=\mathbf{v}\left(t_{1}\right)$ for $t \geq t_{1}$, if $\mathbf{F}(t)=\mathbf{0}$ for $t \geq t_{1}$.

Accounting for $d d t=0$ and $d \mathbf{F}=\mathbf{0}$, one obtains from eq. (1) 


$$
d d\left(\begin{array}{l}
\mathbf{r} \\
\mathbf{v}
\end{array}\right)=\left(\begin{array}{ll}
\mathbf{0} & \mathbf{1} \\
\mathbf{0} & \mathbf{0}
\end{array}\right) d\left(\begin{array}{l}
\mathbf{r} \\
\mathbf{v}
\end{array}\right) d t=\left(\begin{array}{cc}
\mathbf{0} & \mathbf{1} \\
\mathbf{0} & \mathbf{0}
\end{array}\right)\left(\begin{array}{cc}
\mathbf{0} & \mathbf{0} \\
\mathbf{0} & \frac{1}{m} \mathbf{1}
\end{array}\right)\left(\begin{array}{l}
\mathbf{0} \\
\mathbf{F}
\end{array}\right) d t^{2}=\left(\begin{array}{c}
\frac{1}{m} \mathbf{F} \\
\mathbf{0}
\end{array}\right) d t^{2}
$$

Thus, the principles CB1...CB5 are compatible with Newton's equation of motion (published first in [20]). For their relationship to Descartes' and Huygens' principles of motion, see [10][45].

\subsection{Eulerian principles of change of state for Hamiltonian systems}

According to Definition 2 and the axioms, or laws of motion (Laws 1 and 2, Corollary 3), the momentum is the stationary-state variable of a body in Newton's Principia. The total momentum "is not changed by the action of bodies on one another" (Corollary 3). The principles CB1...CB5 remain true, if the velocity, $\mathbf{v}$, is replaced with the momentum, p. For this, I will use $\mathbf{p}$ rather than $\mathbf{v}$ in what follows.

For a free body, any function of the momentum, $Z(\mathbf{p})$, is a conserved quantity. If a body is subject to an external force, its momentum is no longer conserved, but becomes a state-ofmotion variable like its position. Correspondingly, $Z(\mathbf{p}) \neq$ const. Suppose, that there is nevertheless a function, $Z_{0}(\mathbf{p}, \mathbf{r})$, that is constant during the motion of the body. It describes the stationary states of the system body \& force. External influences (additional forces) be described through a function $Z_{e x t}(\mathbf{p}, \mathbf{r}, t)$ such, that $Z(\mathbf{p}, \mathbf{r})=Z_{0}(\mathbf{p}, \mathbf{r})+Z_{e x t}(\mathbf{p}, \mathbf{r}, t)$ takes over the role of the stationary-state function.

The following principles - a generalization of CB1...CB5 - will shown to be compatible with Hamilton's equations of motion. Up to order $d t$,

CS1) The change of stationary-state quantities $(d Z)$ depends solely on the external influences $\left(Z_{\text {ext }}\right)$, but not on state-of-motion quantities $(\mathbf{p}, \mathbf{r})$;

CS2) The change of the stationary-state quantities $(d Z)$ is independent of the stationary-state quantities $(Z)$ themselves;

CS3) The changes of state-of-motion quantities $(d \mathbf{p}, d \mathbf{r})$ directly depend solely on stationarystate quantities $(Z)$; the external influences $\left(Z_{e x t}\right)$ affect the state-of-motion quantities $(\mathbf{p}, \mathbf{r})$ solely indirectly (via stationary-state quantities, $Z$ );

CS4) The changes of stationary-state $(d Z)$ and of state-of-motion quantities $(d \mathbf{p}, d \mathbf{r})$ are independent each of another;

CS5) As soon as the external influences $\left(Z_{\text {ext }}\right)$ vanish, the system remains in the stationary state assumed at this moment: $Z(t)=$ const $=Z\left(t_{1}\right)$ for $t \geq t_{1}$, if $Z_{\text {ext }}=0$ for $t>t_{1}$.

These principles imply the equation of change of stationary state to read

$$
d Z=\frac{\partial Z}{\partial \mathbf{p}} \cdot d \mathbf{p}+\frac{\partial Z}{\partial \mathbf{r}} \cdot d \mathbf{r}+\frac{\partial Z}{\partial t} d t=\frac{\partial Z_{e x t}}{\partial t} d t
$$


This equation is fulfilled, if

$$
\frac{\partial Z}{\partial \mathbf{p}}=a \frac{d \mathbf{r}}{d t} ; \quad \frac{\partial Z}{\partial \mathbf{r}}=-a \frac{d \mathbf{p}}{d t}
$$

Compatibility with Newton's equation of motion yields $a=1$ and $Z(\mathbf{p}, \mathbf{r})=H(\mathbf{p}, \mathbf{r})$, the Hamiltonian of the system; (4) becoming Hamilton's equations of motion.

$$
\frac{d \mathbf{p}}{d t}=-\frac{\partial H}{\partial \mathbf{r}} ; \quad \frac{d \mathbf{r}}{d t}=\frac{\partial H}{\partial \mathbf{p}}
$$

It may thus be not too surprising that these principles can cum grano salis be applied also to quantum systems. Of course, the variables, which represent of stationary states and motion, will be other ones, again.

\section{Quantization as selection problem - I. Derivation of the stationary Schrödinger equation}

The usual foundations of QM consider CM to be not sufficient and, consequently, need additional or novel assumptions, for instance,

- to restrict the energy spectrum to the values $n h v$ [41][6] or to $(n / 2) h v$ [3];

- to "distinguish" [31] or to "select" [39][36] the values $n \hbar$ of the action integral, $\oint_{p d q}(n-$ integer; in contrast to $\mathrm{CM}$, the action integral is not subject to a variational principle);

- to suppose the existence of $h$ and to abandon the classical paths [30];

- to suppose the existence of $h$ and of a wave function being the solution of an eigenvalue problem [43];

- to suppose the existence of a quantum logic [2][28] and of a Hilbert space for its representation;

- to suppose the existence of transition probabilities obeying the Chapman-Kolmogorov equation (as in wave mechanics) [27].

All these approaches have eventually resorted to $\mathrm{CM}$ in using the classical expressions and the interpretations of position, momentum, potential and kinetic energies, because 'it works'. In contrast, I will present a concrete realization of Schrödinger's $4^{\text {th }}$ requirement. 


\subsection{The relationship between $\mathrm{CM}$ and non-CM as selection problem}

In his Nobel Award speech, Schrödinger ([44] p. 315) pointed to the logical aspect, which is central to the approach exposed here.

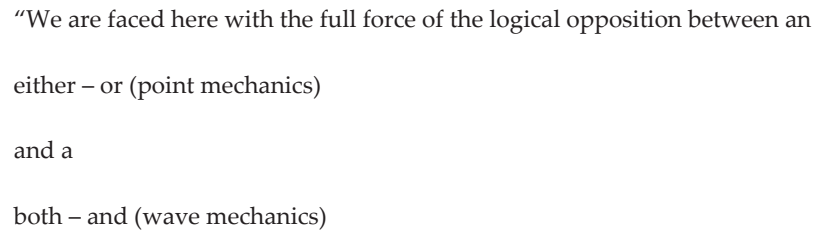

This "logical opposition" consists in a hierarchy of selection problems. ${ }^{1}$

\subsubsection{Selection problem between Newtonian and non-Newtionian CM}

Consider a linear undamped oscillator. For each stationary state of total energy $E$, Newton's equation of motion confines its position, $x$, to the interval between and including the classical turning points: $x_{\min } \leq x \leq x_{\max }$. Its momentum is confined as $p_{\min } \leq p \leq p_{\max }$. More generally speaking, a system obeying Newton's equation of motion moves within the sets $C^{\text {Newton }}=\{\mathbf{r} \mid V(\mathbf{r}) \leq E\}$ and $P^{\text {Newton }}=\{\mathbf{p} \mid T(\mathbf{p}) \leq E\} .^{2}$

Alternatively, a classical, though non-Newtonian mechanics is conceivable, where $d p / d t=-\mathbf{F}$ and $E=V$-T. A linear oscillator would move beyond the turning points: $x \leq x_{\min }$ or/and $x \geq x_{\max }$. In general, the set of possible configurations equals $C^{\text {non-Newton }}=\{\mathbf{r} \mid V(\mathbf{r}) \geq E\}$. The momentum configuration is no longer limited: $P^{\text {non-Newton }}=P^{\text {all }}=\{\mathbf{p}\}$.

Thus, a conservative CM system obeys either the laws of Newtonian CM, where $d \mathbf{p} / d t=+\mathbf{F}$ and $V(\mathbf{r}(t)) \leq E$, or the laws of non-Newtonian CM, where $d \mathbf{p} / d t=-\mathbf{F}$ and $V(\mathbf{r}(t)) \geq E$. In both cases, the system moves along paths, $\mathbf{r}(t)$.

\subsubsection{Einsteinian selection problem between $C M$ and non-CM}

For both Newtonian and non-Newtonian classical systems, the set of possible energies (the energy spectrum) is continuous. Einstein [6] has observed that this leads to a temperatureindependent specific heat (Dulong-Petit's law) for an ensemble of classical oscillators. In contrast, the discrete set of possible energies of a Planck oscillator yields a specific heat that decreases with decreasing temperature, in agreement with then recent experiments. He

\footnotetext{
1 I will deviate from the exposition in [19][10] to make it shorter, though clearer and to correct few statements about the momentum configurations.

$2 \mathrm{~V}(\mathrm{r}) \geq 0$, since it equals the "disposable work storage of a system" [33]. ' $\mathrm{r}$ ' stays for all configuration variables, ' $\mathrm{p}$ ' stays for all momentum configuration variables of a system.
} 
concluded, "that the set of possible energies of microscopic systems is smaller than that for systems of our everyday experience."

Thus, the set of possible energies of a mechanical system is either continuous, or discrete. ${ }^{3}$

\subsubsection{Selection problem between CM and non-CM in terms of allowed configurations}

Einstein's alternative does not follow from purely mechanical arguing. For this, I continue the reasoning of subsection 3.1.1. The harmony between Newtonian and non-Newtonian CMs consists in that they both build an alternative to a non-CM mechanics, in which the set of allowed configurations comprises the whole configuration space, $C^{\text {all }}=C^{\text {Newton }} \cup C^{\text {non-Newton }}=\{\mathbf{r}\}$. Since the motions along paths in $C^{\text {Newton }}$ and $C^{\text {non-Newton }}$ are incompatible each to another, the motion of non-CM systems does not proceed along paths.

Thus, a mechanical system either moves along paths (CM), or it moves not along paths (non$\mathrm{CM}$ ). The configuration of a non-CM system can assume any element of $C^{\text {all }}$ even in the stationary states.

\subsubsection{Selection problem between mechanics and non-mechanics}

For completeness I note that a system is either a mechanical, or a non-mechanical one.

Like Euler's axioms E1 and E2, these alternatives exclude each other and, at once, are "in harmony each with another". They dialectically determine each another in the sense of Hegel [29].

The question thus is how a linear oscillator without turning points is to be described?

\subsection{Non-classical representation of the potential and kinetic energies}

$V(\mathbf{r})[T(\mathbf{p})]$ is no longer the contribution of the (momentum) configuration $\mathbf{r}$ (p) to the total energy, $E$, since it is unbounded in the domain $C^{\text {all }}\left(P^{\text {all }}\right)$. For this, I define 'limiting factors', $F_{E}(\mathbf{r})$ and $G_{E}(\mathbf{p})$, such, that

$$
\begin{aligned}
& V_{E}^{n c l}(\mathbf{r})=F_{E}(\mathbf{r}) V(\mathbf{r}) \leq E ; \quad \mathbf{r} \in C^{\text {all }} \\
& T_{E}^{n c l}(\mathbf{p})=G_{E}(\mathbf{p}) T(\mathbf{p}) \leq E ; \quad \mathbf{p} \in P^{\text {all }}
\end{aligned}
$$

The contribution of the (momentum) configuration $\mathbf{r}(\mathbf{p})$ of a non-classical system to its total energy, $V_{E}{ }^{n c l}(\mathbf{r})\left[T_{E}{ }^{n c l}(\mathbf{p})\right]$, depends on the energy, because the inequality is no longer realized through the restriction of the (momentum) configuration space.

$F_{E}(\mathbf{r})$ and $G_{E}(\mathbf{p})$ are non-negative. $F_{E}(\mathbf{r})<0$ would mean, that $V_{E}{ }^{n c l}(\mathbf{r})$ is attractive (repulsive), while $V(\mathbf{r})$ is repulsive (attractive). $G_{E}(\mathbf{p})<0$ would mean, that $T_{E}{ }^{n c l}(\mathbf{p})$ becomes negative. For simplicity, I chose the one-dimensional representation of unity and set

3 Because of the finite resolution of measurement apparatus, the set of rational numbers is physically equivalent to the set of real numbers. 


$$
F_{E}(\mathbf{r})=\left|f_{E}(\mathbf{r})\right|^{2} ; \quad G_{E}(\mathbf{p})=\left|g_{E}(\mathbf{p})\right|^{2}
$$

" $\psi \Psi\left[\equiv|\psi|^{2}\right]$ is a kind of weight function in the configuration space of the system. The wavemechanical configuration of the system is a superposition of many, strictly speaking, of all kinematically possible point-mechanical configurations. Thereby, each point-mechanical configuration contributes with a certain weight to the true wave-mechanical configuration, the weight of which is just given through $\psi \psi$. If one likes paradoxes, one can say, the system resides quasi in all kinematically thinkable positions at the same time, though not 'equally strongly'. “([43] $4^{\text {th }}$ Commun., p. 135)

Correspondingly, I call $F_{E}$ and $G_{E}$ weight functions, $f_{E}$ and $g_{E}$ - weight amplitudes. Since $F_{E}(\mathbf{r})$ and $G_{E}(\mathbf{p})$ are dimensionless, there are reference values, $r_{\text {ref }}$ and $p_{\text {ref }}$ such, that actually, $F_{E}(\mathbf{r})=F_{E}\left(\mathbf{r} / r_{r e f}\right)$ and $G_{E}(\mathbf{p})=G_{E}\left(\mathbf{p} / p_{r e f}\right)$. In other words, each such system has got a characteristic length in configuration and in momentum configuration space.

$$
\iint_{C^{a l l}} F_{E}\left(\frac{\mathbf{r}}{r_{r e f}}\right) \frac{d^{3} r}{r_{r e f}^{3}}=\iiint_{P^{a l l}} G_{E}\left(\frac{\mathbf{p}}{p_{r e f}}\right) \frac{d^{3} p}{p_{r e f}^{3}}=1
$$

In order to simplify the notation, I will omit $r_{\text {ref }}$ and $p_{\text {ref }}$ wherever possible.

\subsection{The stationary Schrödinger equation}

Within $\mathrm{CM}$, the balance between potential, $V(\mathbf{r})$, and kinetic energies, $T(\mathbf{p})$, to yield $V(\mathbf{r})$ $+T(\mathbf{p})=E=$ const is realized through the common path parameter time, $t: \mathbf{r}=\mathbf{r}(t), \mathbf{p}=\mathbf{p}(t) ; E=V(\mathbf{r}(t))$ $+T(\mathbf{p}(t))$. This common parameterization through $t$ is absent for non-classical systems not moving along paths, $\mathbf{r}(t)$. Consequently, the balance between the potential, $V_{E}{ }^{n c l}(\mathbf{r})$, and kinetic energies, $T_{E}{ }^{n c l}(\mathbf{p})$, is not point-wise: $\mathbf{p}(t) \leftrightarrow \mathbf{r}(t)$, but set-wise: $\{\mathbf{p}\} \leftrightarrow\{\mathbf{r}\}$. Set-wise relations are mediated through integral relations. ${ }^{4}$

$$
g_{E}(\mathbf{p})=\frac{1}{\left(2 \pi p_{r e f} r_{r e f}\right)^{\frac{3}{2}}} \iint_{C^{a l l}} e^{i \frac{\mathbf{p} \cdot \mathbf{r}}{p_{r e f} r_{r e f}}} f_{E}(\mathbf{r}) d^{3} r ; \quad f_{E}(\mathbf{r})=\frac{1}{\left(2 \pi p_{r e f} r_{r e f}\right)^{\frac{3}{2}}} \iiint_{P^{a l l}} e^{-i \frac{\mathbf{r} \cdot \mathbf{p}}{p_{r e f} r_{r e f}}} g_{E}(\mathbf{p}) d^{3} p
$$

In view of the symmetric normalization (8) I have chosen symmetric normalization factors.

Alternatively, it is possible to avoid complex-valued weight amplitudes (wave functions) through using 2-component vectors for them and the Hartley transform in place of the Fourier

4 The most general symmetric Fourier transform contains a free complex-valued parameter [49]. It appears to be merely a rescaling of $\mathrm{r}_{\text {ref }}$ and $\mathrm{p}_{\text {ref }}$ respectively. 
transform. The operators become $2 \times 2$ matrices. It remains to explore whether their free components can be exploited for the description of new effects.

Lacking orbits, such a system does not assume a definite configuration, say, $\mathbf{r}_{1}$, and momentum configuration, $\mathbf{p}_{1}$, at a given time, $t_{1}$, with $E=V_{E}{ }^{n c l}\left(\mathbf{r}_{1}\right)+T_{E}{ }^{n c l}\left(\mathbf{p}_{1}\right)$. Instead, they all contribute to the stationary state, $E$. The partial contribution of the single (momentum) configuration, $\mathbf{r}(\mathbf{p})$, is determined by the weight function according to eq. (6). The total energy thus becomes

$$
E=\frac{\iiint_{C^{\text {all }}} F_{E}(\mathbf{r}) V(\mathbf{r}) d^{3} r}{\iiint_{C^{\text {all }}} F_{E}(\mathbf{r}) d^{3} r}+\frac{\iiint_{P^{a l l}} G_{E}(\mathbf{p}) T(\mathbf{p}) d^{3} p}{\iiint_{P^{a l l}} G_{E}(\mathbf{p}) d^{3} p}
$$

The denominators have been added for dimensional reasons. The classical representation is obtained through setting

$$
F_{E}(\mathbf{r})=r_{r e f}^{3} \delta(\mathbf{r}-\mathbf{r}(t)) ; \quad G_{E}(\mathbf{p})=p_{r e f}^{3} \delta(\mathbf{p}-\mathbf{p}(t))
$$

The occurrence of $E$ on the r.h.s. makes eq. (10) to be an implicit equation for $E$. This suggests $E$ to be an internal system parameter being determined solely by system properties like the oscillation frequency of an undamped harmonic oscillator [19]. However, as in CM, the value of $E$ is given by the initial preparation of the system.

The Fourier transform (9) enables me to eliminate one of the weight amplitudes from eq. (10).

$$
\begin{aligned}
& E=\frac{\iiint_{C^{a l l}} \bar{f}_{E}(\mathbf{r}) \hat{H}(\mathbf{r}) f_{E}(\mathbf{r}) d^{3} r}{\iiint_{C^{\text {all }}} \bar{f}_{E}(\mathbf{r}) f_{E}(\mathbf{r}) d^{3} r} ; \quad \hat{H}(\mathbf{r}) \equiv V(\mathbf{r})+T\left(-i p_{r e f} r_{r e f} \frac{\partial}{\partial \mathbf{r}}\right) \\
& =\frac{\iiint_{P^{a l l}} \bar{g}_{E}(\mathbf{p}) \hat{H}(\mathbf{p}) g_{E}(\mathbf{p}) d^{3} p}{\iiint_{P^{\text {all }}} \bar{g}_{E}(\mathbf{p}) g_{E}(\mathbf{p}) d^{3} p} ; \quad \hat{H}(\mathbf{p}) \equiv V\left(i r_{r e f} p_{r e f} \frac{\partial}{\partial \mathbf{p}}\right)+T(\mathbf{p})
\end{aligned}
$$

(Other positions of the weight amplitudes lead to the same results.) Since, in general, $\bar{f}_{E}$ and $\bar{g}_{E}$ are linearly independent of $f_{E}$ and $g_{E}$, respectively, necessary conditions for fulfilling these equations are

$$
E f_{E}(\mathbf{r})=\hat{H}(\mathbf{r}) f_{E}(\mathbf{r}) ; \quad E g_{E}(\mathbf{p})=\hat{H}(\mathbf{p}) g_{E}(\mathbf{p})
$$


Moreover, these equations hold true for the minimum of the r.h.s. of eq. (12), ie, for the ground state. There is no indication for a difference between the stationary-state equation for the ground state and for the states of higher energy.

A comparison with experiments reveals, that $r_{r e f} p_{r e f}=\hbar$, which I will use in what follows. Thus, with $f_{E}(r)=r_{r e f}^{3 / 2} \psi_{E}(r)$ and $g_{E}(p)=p_{r e f}^{3 / 2} \phi_{E}(p)$, eqs. (13) are the stationary Schrödinger equations in configuration and momentum configuration spaces.

\section{Quantization as selection problem - II. Non-classical solution to the stationary Schrödinger equation}

As observed by Schrödinger himself (!), the eigenvalue method used by himself does not properly account for the quantum nature of quantum systems, because it applies to (and had been developed for) classical systems like strings and pipes. In what follows, I will describe a solution method being free of that deficiency.

\subsection{The linear oscillator}

The stationary Schrödinger equation for a linear undamped harmonic oscillator reads

$$
\hat{H}(x) f_{E}\left(\frac{x}{r_{r e f}}\right)=E f_{E}\left(\frac{x}{r_{r e f}}\right) ; \quad \hat{H}(x)=\frac{m}{2} \omega^{2} x^{2}-\frac{\hbar^{2}}{2 m} \frac{\partial^{2}}{\partial x^{2}}
$$

To see its essentials I introduce dimensionless variables as ${ }^{5}$

$$
\xi=\frac{x}{r_{r e f}} ; \quad r_{r e f}=\sqrt{\frac{r_{r e f} p_{r e f}}{2 m \omega}} ; \quad y_{a}(\xi)=f_{E}(\xi) ; \quad a=-\frac{E}{r_{r e f} p_{r e f} \omega}
$$

to obtain

$$
\frac{d^{2} y_{a}(\xi)}{d \xi^{2}}-\left(\frac{1}{4} \xi^{2}+a\right) y_{a}(\xi)=0
$$

This is Weber's equation [48] being one of the equations of the parabolic cylinder [1]. Despite of the reference length, $r_{\text {ref }}$ the stationary states are determined solely through the energy parameter, $-a$. In contrast to the classical oscillator, where $E \sim \omega^{2}$, the quantum oscillator exhibits

5 This yields $\mathrm{p}_{\mathrm{ref}}=2 \mathrm{~m} \omega \mathrm{r}_{\mathrm{ref}}$; the classical maximum values are interrelated as $\mathrm{p}_{\max }=\mathrm{m} \omega \mathrm{r}_{\max }$. I deviate from the exposition in [19][10] to make the following clearer. 
$E \sim \omega$. Since $\omega$ does not occur as a self-standing parameter, the quantization is not affecting it; Schrödinger's $3^{\text {rd }}$ requirement is fulfilled.

\subsection{The mathematically distinguished solutions}

For and only for the values $a= \pm 1 / 2$ the l.h.s. of eq. (16) factorizes. ${ }^{6}$

$$
\left(\frac{d}{d \xi}+\frac{1}{2} \xi\right)\left(\frac{d}{d \xi}-\frac{1}{2} \xi\right) y_{+1 / 2}(\xi)=0 ; \quad\left(\frac{d}{d \xi}-\frac{1}{2} \xi\right)\left(\frac{d}{d \xi}+\frac{1}{2} \xi\right) y_{-1 / 2}(\xi)=0
$$

Therefore, the values $a= \pm 1 / 2$ are mathematically distinguished against all other $a$-values. The corresponding solutions, $y_{ \pm 1 / 2}(\xi)$, are mathematically equivalent, but physically different. $y_{-1 / 2}$ $(\xi)=y_{-1 / 2}(0) \times \exp \left(-\xi^{2} / 4\right)$ is a limiting amplitude, while $y_{+1 / 2}(\xi)=y_{+1 / 2}(0) \times \exp \left(+\xi^{2} / 4\right)$ is not. This distinguishes physically the value $a=-1 / 2$ over the value $a=+1 / 2$.

If there would be no other distinguished $a$-values, there would be only one state $(a=-1 / 2)$. However, a system having got just one state is not able to exchange energy with its environment. In order to find further distinguished $a$-values, I examine two recurrence relations for the standard solutions of eq. (16) ([1] 19.6.1, 19.6.5).

$$
\left(\frac{d}{d \xi}+\frac{1}{2} \xi\right) U(a, \xi)+\left(a+\frac{1}{2}\right) U(a+1, \xi)=0 ; \quad\left(\frac{d}{d \xi}-\frac{1}{2} \xi\right) V(a, \xi)-\left(a-\frac{1}{2}\right) V(a-1, \xi)=0
$$

Such recurrence relations can be obtained without solving the differential equation, viz, from Whittaker's representation of the solutions as contour integrals ([52] 16.61; [1] 19.5). This representation has been developed well before the advance of QM; it is thus independent of the needs of QM.

The recurrence relations

- are not related to the usual, classical solution methods;

- interrelate solution functions with a finite difference between their $a$-values, viz, $\Delta a= \pm 1$ (this becomes $\Delta E= \pm \hbar \omega$ later on);

- reflect the genuine discrete structure wanted; in particular, this structure has nothing to do with boundary conditions, since all solutions exhibit this discrete structure, not only Schrödinger's eigensolutions;

- realize the "conviction", that "the true laws of quantum mechanics would consist not of specific prescriptions for the single orbit; but, in these true laws, the elements of the whole manifold of orbits of a system are connected by equations, so that there is apparently a certain interaction between the various orbits." ([43] Second Commun., p. 508)

6 These factors are closely related to the variables that factorize the classical Hamiltonian. 
Moreover, the recurrence relations divide the set of $a$-values as follows.

Set (1) $a=\ldots,-5 / 2,-3 / 2,-1 / 2$; the $2^{\text {nd }}$ relation (18) breaks at $a=-1 / 2$ being one of the two mathematically distinguished values found above;

Set (2) $a=\ldots, 5 / 2,3 / 2,1 / 2$; the $1^{\text {st }}$ relation (18) breaks at $a=+1 / 2$ being the other mathematically distinguished value found above;

Set (3) $a=\{\ldots,-2+\varsigma,-1+\varsigma, \varsigma, \varsigma+1, \varsigma+2 \ldots \mid-1 / 2<\varsigma<+1 / 2\}$; there is no break in the recurrence relations (18) for this set.

The smallest interval representing all solutions is the closed interval $a=[-1 / 2,+1 / 2]$, all other solutions being related to it through the recursion formulae. The values $a=-1 / 2$ (set (1)) and $a=$ $+1 / 2$ (set (2)) are mathematically distinguished, again; this time as the boundary points of that interval. All inner interval points, $-1 / 2<a<+1 / 2(\operatorname{set}(3))$, are mathematically equivalent among each another and, consequently, not distinguished. The physically relevant set of $a$-values is a mathematically distinguished set.

\subsection{The physically distinguished solutions}

The mathematically distinguished set (1) contains the physically relevant value $a=-1 / 2$, while the mathematically distinguished set (2) contains the unphysical value $a=+1 / 2$. The recurrence relations (18) show, that all functions $U(a, \xi)$ with $a$-values from set (1) are limiting amplitudes, while all functions $V(a, \xi)$ with $a$-values from set (2) are not. For the $a$-values of set (3), neither $U(a, \xi)$, nor $V(a, \xi)$ is a limiting amplitude.

Moreover, set (1) exhibits a finite minimum of total energy, $E=1 / 2 r_{\text {ref }} p_{\text {ref }} \omega$, while sets (2) and (3) do not. A system of sets (2) or (3) can deliver an unlimited amount of energy to its environment, it is a perpetuum mobile of $1^{\text {st }}$ kind. This makes set (1) to be physically distinguished against sets (2) and (3).

Hence, starting from the ground state, $y_{-1 / 2}(\xi)=y_{-1 / 2}(0) \times \exp \left(-\xi^{2} / 4\right)$, and using the recursion formula (18) for $U(a, \xi)$, the physically relevant solutions are obtained as

$$
y_{-n-\frac{1}{2}}(\xi)=e^{-\frac{1}{4} \xi^{2}} H e_{n}(\xi) ; \quad n=0,1,2, \ldots
$$

where

$$
H e_{n}(\xi)=(-1)^{n} e^{\frac{1}{2} \xi^{2}} \frac{d^{n}}{d \xi^{n}} e^{-\frac{1}{2} \xi^{2}}
$$

is the $n^{\text {th }}$ Hermite polynomial ([1] 19.13.1). Schrödinger's boundary condition (the wave function should vanish at infinity) is fulfilled automatically.

Since ([1] 22.2) 


$$
\int_{-\infty}^{+\infty} e^{-\frac{1}{2} \xi^{2}} H e_{n}(\xi)^{2} d \xi=\sqrt{2 \pi} n !
$$

the normalized solutions [see eq. (8)] read

$$
y_{-n-\frac{1}{2}}(\xi)=\frac{1}{\sqrt{\sqrt{2 \pi} n !}} e^{-\frac{1}{4} \xi^{2}} H e_{n}(\xi) ; \quad n=0,1,2, \ldots
$$

\subsection{The non-classical potential energy and the tunnel effect}

The observation of quantum particles crossing spatial domains, where $V>E$, has led to the notion 'tunnel effect' [34][38]. Being a nice illustration, this wording masks the fact, that the actual contribution of a configuration, $\mathbf{r}$, to the total energy is not $V(\mathbf{r})$, but $V_{E}{ }^{n c l}(\mathbf{r})<E$, see eq. (6). In terms of the dimensionless variables (15) the dimensionless non-classical potential energy of the oscillator above equals

$$
v_{n}^{n c l}(\xi)=y_{-n-\frac{1}{2}}^{2}(\xi) \frac{1}{2} \xi^{2}=\frac{1}{2 \sqrt{2 \pi} n} \xi^{2} e^{-\frac{1}{2} \xi^{2}} H e_{n}(\xi)^{2}
$$

Using the recurrence formula $\xi H e_{n}(\xi)=H e_{n+1}(\xi)+n H e_{n-1}(\xi)$ ([1] 22.7.14) and the inequality I $\mathrm{He}_{n}(\xi) \mid<\exp \left(\xi^{2} / 4\right) \sqrt{ }(n !) k, k \approx 1.086435$ (ibid., 22.14.17), one can prove, that

$$
v_{n}^{n c l}(\xi)=y_{-n-\frac{1}{2}}(\xi)^{2} \frac{1}{2} \xi^{2}<n+\frac{1}{2} ; \quad-\infty<\xi<+\infty ; \quad n=0,1,2, \ldots
$$

Hence, the inequalities (6) are fulfilled.

The occurrence of the 'smaller than' sign means, that - in contrast to the classical oscillator there are no stationary states with, (i), vanishing potential energy (in particular, the ground state is not a state at rest) and, (ii) vanishing kinetic energy (there are no turning points).

The picture of the tunnel is partially correct, in that the classical turning points are points of inflection such, that beyond them, in the forbidden domains of Newtonian CM, the wave function decreases exponentially.

Notice that these results follow solely from the most general principles of state description according to Leibniz [35], Euler, Helmholtz and Schrödinger, without solving any stationarystate equation or equation of motion and without assuming particular boundary conditions. 


\section{The time dependent case}

While Heisenberg [30] and Schrödinger [43] started from a time-dependent equation ${ }^{7}$, I have worked so far with the set of all possible (momentum) configurations of systems in their stationary states, where time plays no role. In order to incorporate time, I will proceed as Newton and Euler did in the classical case and will consider first the time-dependence of the stationary states, then, the change of these states, and, finally, I will arrive at the timedependent Schrödinger equation as the equation of motion.

\subsection{The time dependence of the stationary states}

According to their definitions (6), the stationary weight functions, $F_{E}(\mathbf{r})$ and $G_{E}(\mathbf{p})$, are time independent. Hence, if there is a time dependence of the stationary weight amplitudes, and correspondingly of the wave functions, it is of the form

$$
\psi_{E}(\mathbf{r}, t)=e^{i \phi_{E}(t)} \psi_{E}(\mathbf{r}) ; \quad \varphi_{E}(\mathbf{p}, t)=e^{i \phi_{E}(t)} \varphi_{E}(\mathbf{p})
$$

The phase, $\varphi_{E}(t)$, is the same for both functions, since the Fourier transform (9) is timeindependent.

For a free particle,

$$
\psi_{E}(\mathbf{r}, t) \sim \exp \left\{i \mathbf{k}_{E} \cdot \mathbf{r}-i \omega_{E} t\right\} ; \quad E=\frac{\hbar^{2} k_{E}^{2}}{2 m}
$$

The group velocity equals the time-independent particle velocity.

$$
\mathbf{v}_{g}=\frac{d \omega_{E}}{d \mathbf{k}_{E}}=\frac{\hbar \mathbf{k}_{E}}{m} \Rightarrow \omega_{E}=\frac{\hbar k_{E}^{2}}{2 m}=\frac{E}{\hbar}
$$

Therefore,

$$
\psi_{E}(\mathbf{r}, t)=e^{-i \frac{E}{\hbar} t} \psi_{E}(\mathbf{r}) ; \quad \phi_{E}(\mathbf{p}, t)=e^{-i \frac{E}{\hbar} t} \phi_{E}(\mathbf{p})
$$

For later use I remark that this can be written as

$7 \mathrm{In} \mathrm{fn.} \mathrm{2,} \mathrm{p.} \mathrm{489,} \mathrm{of} \mathrm{the} 2^{\text {nd }}$ Commun., Schrödinger has distanced himself from the time-independent approach of the $1^{\text {st }}$ Commun. 


$$
\psi_{E}(\mathbf{r}, t)=e^{-\frac{i}{\hbar} \hat{H}(\mathbf{r}) t} \psi_{E}(\mathbf{r}) ; \quad \phi_{E}(\mathbf{p}, t)=e^{-\frac{i}{\hbar} \hat{H}(\mathbf{p}) t} \phi_{E}(\mathbf{p})
$$

\subsection{The equation-of-state-change}

The analog to the Hamiltonian as classical stationary-state function is the function

$$
Z^{n c l}=\frac{\iiint_{C^{a l l}} \bar{f}(\mathbf{r}, t) \hat{H}(\mathbf{r}, t) f(\mathbf{r}, t) d^{3} r}{\iiint_{C^{a l l}} \bar{f}(\mathbf{r}, t) f(\mathbf{r}, t) d^{3} r}=\frac{\iiint_{p^{a l l}} \bar{g}(\mathbf{p}, t) \hat{H}(\mathbf{p}, t) g(\mathbf{p}, t) d^{3} p}{\iiint_{P^{a l l}} \bar{g}(\mathbf{p}, t) g(\mathbf{p}, t) d^{3} p}
$$

The analog to the classical principles of change of state, CS1...CS5, reads as follows. Up to first order in $d t$,

QS1) the change of stationary-state quantities $(d Z)$ depends solely on the external causes $\left(Z_{e x t}\right)$, but not on state-of-motion quantities $(f, g)$;

QS2) the changes of the stationary-state quantities $(d Z)$ are independent of the stationary-state quantities $(Z)$ themselves;

QS3) the changes of state-of-motion quantities $(d f, d g)$ depend directly solely on stationarystate quantities $(Z)$;

QS4) the changes of stationary-state $(d Z)$ and of state-of-motion quantities ( $d f, d g)$ are independent each of another;

QS5) as soon as the external causes $\left(Z_{e x t}\right)$ vanish, the system remains in the (not necessarily stationary $^{8}$ ) state assumed in this moment.

Hence, writing

$$
\iiint \bar{f}(\mathbf{r}, t) \hat{H}(\mathbf{r}, t) f(\mathbf{r}, t) d^{3} r=\langle f(\mathbf{r}, t)|\hat{H}(\mathbf{r}, t)| f(\mathbf{r}, t)\rangle
$$

the equation of state change becomes

$$
\begin{aligned}
d Z^{n c l} & =\frac{\langle d f(\mathbf{r}, t)|\hat{H}(\mathbf{r}, t)| f(\mathbf{r}, t)\rangle+\langle f(\mathbf{r}, t)|d \hat{H}(\mathbf{r}, t)| f(\mathbf{r}, t)\rangle+\langle f(\mathbf{r}, t)|\hat{H}(\mathbf{r}, t)| d f(\mathbf{r}, t)\rangle}{\langle f(\mathbf{r}, t) \mid f(\mathbf{r}, t)\rangle} \\
& -\frac{\langle d f(\mathbf{r}, t) \mid f(\mathbf{r}, t)\rangle+\langle f(\mathbf{r}, t) \mid d f(\mathbf{r}, t)\rangle}{\langle f(\mathbf{r}, t) \mid f(\mathbf{r}, t)\rangle} \frac{\langle f(\mathbf{r}, t)|\hat{H}(\mathbf{r}, t)| f(\mathbf{r}, t)\rangle}{\langle f(\mathbf{r}, t) \mid f(\mathbf{r}, t)\rangle} \\
& !\langle f(\mathbf{r}, t)|d \hat{H}(\mathbf{r}, t)| f(\mathbf{r}, t)\rangle \\
\langle f(\mathbf{r}, t) \mid f(\mathbf{r}, t)\rangle &
\end{aligned}
$$

8 The modification against CS5 is a consequence of the discreteness of the energetic spectrum. 


\subsection{Derivation of the time-dependent Schrödinger equation}

The requirement in eq. (32) implies two conditions.

$$
\begin{aligned}
& \langle d f(\mathbf{r}, t)|\hat{H}(\mathbf{r}, t)| f(\mathbf{r}, t)\rangle+\langle f(\mathbf{r}, t)|\hat{H}(\mathbf{r}, t)| d f(\mathbf{r}, t)\rangle=0 \\
& \langle d f(\mathbf{r}, t) \mid f(\mathbf{r}, t)\rangle+\langle f(\mathbf{r}, t) \mid d f(\mathbf{r}, t)\rangle=d\langle f(\mathbf{r}, t) \mid f(\mathbf{r}, t)\rangle=0
\end{aligned}
$$

The second condition means that there is a unitary time development operator,

$$
\hat{U}\left(\mathbf{r}, t_{2}, t_{1}\right) f\left(\mathbf{r}, t_{1}\right)=f\left(\mathbf{r}, t_{2}\right) ; \quad \hat{U}\left(\mathbf{r}, t_{2}, t_{1}\right)^{\dagger}=\hat{U}\left(\mathbf{r}, t_{2}, t_{1}\right)^{-1}
$$

such, that

$$
\left\langle f\left(\mathbf{r}, t_{2}\right) \mid f\left(\mathbf{r}, t_{2}\right)\right\rangle=\left\langle\hat{U}\left(\mathbf{r}, t_{2}, t_{1}\right) f\left(\mathbf{r}, t_{1}\right) \mid \hat{U}\left(\mathbf{r}, t_{2}, t_{1}\right) f\left(\mathbf{r}, t_{1}\right)\right\rangle=\left\langle f\left(\mathbf{r}, t_{1}\right) \mid f\left(\mathbf{r}, t_{1}\right)\right\rangle
$$

Now I insert eq. (34) into the first requirement (33).

$$
\langle d \hat{U}(\mathbf{r}, t, 0) f(\mathbf{r}, 0)|\hat{H}(\mathbf{r}, t)| \hat{U}(\mathbf{r}, t, 0) f(\mathbf{r}, 0)\rangle+\langle\hat{U}(\mathbf{r}, t, 0) f(\mathbf{r}, 0)|\hat{H}(\mathbf{r}, t)| d \hat{U}(\mathbf{r}, t, 0) f(\mathbf{r}, 0)\rangle=0
$$

The unitary solution to this equation reads $d \hat{U}(r, t, 0)=j u(\hat{H}(r, t)) d t$, where $u(\hat{H})$ is a realvalued rational function of the self-adjoint Hamiltonian, $H$. Compatibility with the stationary case (29) yields $u(\hat{H})=-\frac{1}{\hbar} \hat{H}$. Hence,

$$
\hat{U}(\mathbf{r}, t, 0)=\hat{P}\left(\exp \left\{\frac{-i}{\hbar} \int_{0}^{t} \hat{H}\left(\mathbf{r}, t^{\prime}\right) d t^{\prime}\right\}\right)
$$

where $\hat{P}$ denotes Dyson's time-ordering operator [4]. The time-dependent Schrödinger equation for $f(r, t)$ follows immediately.

The momentum representation can be derived quite analogously.

Both representations of the time-dependent Schrödinger form two equivalent equations of motion. As in the classical case, the equation of motion is a dynamic equation for nonstationary-state entities.

\section{Summary and conclusions}

I have presented a relatively novel approach to quantization, viz, quantization as selection rather than eigenvalue problem. It starts from Euler's rather than Newton's axiomatic and exploits Helmholtz's [32][33] treatment of the energy conservation law. It fulfills all four of Schrödinger's methodical requirements quoted in the Introduction. 
It is often assumed, that the difference between classical and quantum systems is caused by the existence of the quantum of action. I have shown that this assumption is not necessary. It is sufficient to make different assumptions about the set of (momentum) configurations a mechanical system can assume in its stationary states.

The "logical opposition" between CM and QM observed by Schrödinger [44] is actually a dialectic relationship, which resembles that between the Finite and the Infinite. Each determination draws a Limit, where each Limit involves the existence of something beyond it ( $f$ [29] Logik I p. 145). The notion of the Finite does not exist without the notion of the Infinite (ibid. pp. 139ff.). The Infinite is the Other of the Finite - in turn, the Finite is the Other of the Infinite. Now, the Finite and the Infinite are not simply opposites; a border between them would contradict the very meaning of infinity. The True Infinite includes the Finite, it is the unity of the Finite and the Infinite (cfibid. p. 158).

The solution of the stationary Schrödinger equation without using boundary conditions shows, that it actually does "carry the quantum conditions in itself" (Schrödinger's ${ }^{\text {st }}$ requirement, see Introduction). Hence, it has got "maximum strength" in the sense of Einstein [8].

$\mathrm{CM}$ contains the necessary means for going beyond its own frame. This way, the relationship between $\mathrm{CM}$ and non-CM becomes well defined, and the physical content of non-CM is formulated on equal footing with the mathematical method (and vice versa). An example for this is the reformulation of Einstein's criterion (the number of stationary states) in terms of recurrence relations.

Ad-hoc assumptions, which may be suggested by experimental results, but are not supported by the axiomatic of $\mathrm{CM}$, can be avoided. The wave and particle aspects can be obtained from the time-dependent Schrödinger equation and its solutions [10]. The classical path in phase space is replaced with the wave functions in space and momentum representations. The wave functions take also the role of the initial conditions, which "are not free, but also have to obey certain laws" [7].

The dynamics in space and in momentum space are treated in parallel. As a consequence, the Schrödinger equation in momentum representation is obtained at once with the one in position representation. This, too, enables one to keep maximum contact to $\mathrm{CM}$ and to explain, why $\mathrm{QM}$ is a non-classical mechanics of conservative systems, where the classical potential and kinetic energy functions and, consequently, the classical Lagrange and Hamilton functions still apply. This includes a natural explanation of "the peculiar significance of the energy in quantum mechanics" [51].

Modern representations of $\mathrm{CM}$ favor equations of motion as the foundation (the variational principles belong to this class). The state variables are position and velocity (Lagrange, Laplace), or position and momentum (Hamilton). Hence, there are 6 state variables for a single body. In contrast, there are only 3 quantum numbers for a spinless particle. And there are only 3 stationary-state variables for a single body within Newton's (the 3 components of the momentum vector) and Euler's (the 3 components of the velocity vector) representations of $\mathrm{CM}$, respectively. ${ }^{9}$ This is another indication for the fact, that the latter are more suitable for the transition from CM to QM than Lagrange's and Hamilton's representations. 
For the quantization of fields, finally, this approach yields an explanation for the fact, that, within the method of normal-mode expansion, only the temporal, but not the spatial part of the field variables is concerned ( $c f$ [42]). Indeed, only those variables are subject to the quantization procedure, the possible values of which are restricted by the energy law. The spatial extension of the normal modes is fixed by the boundary conditions and thus not subject to quantization. The classical field energy (density) is determined by the normal-mode amplitudes and thus limits these. As a consequence, the time-dependent coefficients in the normal-mode expansion are quantized. When formulating this expansion such, that these expansion coefficients get the dimension of length, their quantization can be performed in complete analogy to that of the harmonic oscillator, without invoking additional assumptions or new constants [10]. Moreover, one could try to quantize a field in the space spanned by independent dynamical field variables. This could separate the quantization problem from the spatial and temporal field distributions and, thus, simplify the realization of Einstein's imagination of a "spatially granular" [5] structure of the electromagnetic field.

\title{
Acknowledgements
}

I feel highly indebted to Dr. D. Suisky with whom the basic ideas of 'quantization as selection problem' have been elaborated [46][47][18][19]. Over the years I have benefited from numerous discussions with Dr. M. Altaisky, Prof. Y. Dabaghian, Dr. M. Daumer, Dr. K. Ellmer, Dr. D. B. Fairlie, Dr. A. Förster, Prof. L. Fritsche, Prof. W. Greiner, Dr. H. Hecht, H. Hille, Prof. J. Keller, Prof. J. R. Klauder, Prof. H. Kröger, Dr. Th. Krüger, Prof. H. Lübbig, Prof. G. Mann, Prof. Matone, Prof. S. N. Mayburow, Prof. P. Mittelstaedt, Dr. R. Müller, Prof. J. G. Muroz, Prof. G. Nimtz, Prof. H. Paul, Prof. Th. Pöschel, Prof. J. Rosen, A. Rothenberg, Prof. W. P. Schleich, Prof. J. Schröter, Prof. J. Schnakenberg, Prof. J. Schröter, Dr. W. Smilga, Dr. E. V. Stefanovich, Dr. L. Teufel, Dr. R. Tomulka, Prof. H. Tributsch, Dr. M. Vogt, Prof. R. F. Werner and many more. I am indebted to Profs. M. Müller-Preußker and W. Nolting for their continuous interest and support. Early stages of this work were supported by the Deutsche Akademie der Naturforscher Leopoldina [18], Prof. Th. Elsässer and Prof. E. Siegmund.

\section{Author details}

\author{
Peter Enders*
}

Address all correspondence to: enders@dekasges.de

University of Applied Sciences, Wildau, Königs Wusterhausen, Germany

9 Some implications of this similarity between Newton's and Euler's notions of state on the classical and Schrödinger's and Pauli's [39][40] on the quantum sides have been investigated in [9][11][12][15][16]. 


\section{References}

[1] Abramowitz, M, \& Stegun, I. A. Eds.), Handbook of Mathematical Functions, Washington: NBS 1964; abbreviated reprint (selection by M. Danos \& J. Rafelski): Pocketbook of Mathematical Functions, Thun Frankfurt/Main: Deutsch (1984).

[2] Birkhoff, G, \& Von Neumann, J. The logic of quantum mechanics, Ann. of Math. [2] (1936). , 37(1936), 823-843.

[3] Bohr, N. (1913). On the Constitution of Atoms and Molecules", Phil. Mag. , 26, 1-13.

[4] Dyson, F. J. (1949). The radiation theories of Tomonaga, Schwinger, and Feynman", Phys. Rev. , 75, 486-502.

[5] Einstein, A. (1905). Über einen die Erzeugung und Verwandlung des Lichtes betreffenden heuristischen Gesichtspunkt", Ann. Phys. , 17, 132-148.

[6] Einstein, A. (1907). Die Plancksche Theorie der Strahlung und die Theorie der spezifischen Wärme", Ann. Phys. 22, , $180 \mathrm{ff}$.

[7] Einstein, A. (1923). Bietet die Feldtheorie Möglichkeiten für die Lösung des Quantenproblems?", Sitzungsber. Preuss. Ak. Wiss. phys.-math. K1., 13. Dez., XXXIII, , $359 \mathrm{ff.}$

[8] Einstein, A. (1977). Grundzüge der Relativitätstheorie (Akademie-Verlag, Berlin), , 132.

[9] Enders, P. (2004). Equality and Identity and (In)distinguishability in Classical and Quantum Mechanics from the Point of View of Newton's Notion of State, $6^{\text {th }}$ Int. Symp. Frontiers of Fundamental and Computational Physics, Udine; in: Sidharth, Honsell \& De Angelis (Hrsg.), Frontiers of Fundamental Physics, 2006, , 239-245.

[10] Enders, P. (2006). Von der klassischen Physik zur Quantenphysik. Eine historisch-kritische deduktive Ableitung mit Anwendungsbeispielen aus der Festkörperphysik, Berlin Heidelberg: Springer

[11] Enders, P. Is Classical Statistical Mechanics Self-Consistent? (A paper of honour of C. F. von Weizsäcker, 1912-2007), Progr. Phys. (2007). http://www.allbusiness.com/ science-technology/physics/5518225-1.html, 3(2007), 85-87.

[12] Enders, P. Equality and Identity and (In)distinguishability in Classical and Quantum Mechanics from the Point of View of Newton's Notion of State, Icfai Univ. J. Phys. I ((2008). http://www.iupindia.org/108/IJP_Classical_and_Quantum_Mechanics_71.html

[13] Enders, P. Towards the Unity of Classical Physics, Apeiron 16 ((2009). http:// redshift.vif.com/JournalFiles/V16N1END.pdf

[14] Enders, P. Huygens principle as universal model of propagation, Latin Am. J. Phys. Educ. (2009). http://dialnet.unirioja.es/servlet/articulo?codigo=3688899, 3(2009), $19-32$.

[15] Enders, P. Gibbs' Paradox in the Light of Newton's Notion of State, Entropy (2009). http:// www.mdpi.com/1099-4300/11/3/454, 11(2009), 454-456. 
[16] Enders, P. State, Statistics and Quantization in Einstein's 1907 Paper, 'Planck's Theory of Radiation and the Theory of Specific Heat of Solids', Icfai Univ. J. Phys. II ((2009). http:// www.iupindia.org/709/IJP_Einsteins_1907_Paper_176.html

[17] Enders, P. (2010). Precursors of force fields in Newton's 'Principia'", Apeiron 17, 22-27; http://redshift.vif.com/JournalFiles/V17N1END.PDF

[18] Enders, P, \& Suisky, D. (2004). Über das Auswahlproblem in der klassischen Mechanik und in der Quantenmechanik", Nova Acta Leopoldina, Suppl. , 18, 13-17.

[19] Enders, P, \& Suisky, D. (2005). Quantization as selection problem", Int. J. Theor. Phys. , 44, 161-194.

[20] Euler, L. II-1, "Mechanica sive motus scientia analytice exposita", in: Leonardi Euleri Opera Omnia sub auspiciis Societatis Scientarium Naturalium Helveticae (Zürich Basel, 1911 - 1986), ser. II, vol. 1

[21] Euler, L, \& Découverte, I. I-5a. d'une nouveau principe de mécanique", in: Opera Omnia, ser. II, , 5

[22] Euler, L. II-5b, "Harmonie entre les principes généraux de repos et de mouvement de M. de Maupertuis", in: Opera Omnia, ser. II, , 5

[23] Euler, L. II-5c, "Recherches sur l'origine des forces", Mém. ac. sci. Berlin 6 (1750) 1752, 419-447; in: Opera Omnia, ser. II, , 5

[24] Euler, L. III-1, "Anleitung zur Naturlehre", in: Opera Omnia, ser. III, , 1

[25] Euler, L. III-11, "Lettres à une princesse d'Allemagne sur divers sujets de Physique et de Philosophie", in: Opera Omnia, ser. III, vols. 11 and 12

[26] Faraggi, A. E, \& Matone, M. (1998). The Equivalence Postulate of Quantum Mechanics", arXiv:hep-th/9809127

[27] Feynman, R. P. (1949). Space-Time Approach to Quantum Electrodynamics", Phys. Rev., 76, , 769ff.

[28] FrancisCh. (2010). Quantum Logic, http://rqgravity.net/FoundationsOfQuantumTheory\#QuantumLogic

[29] (G. F. W. Hegel, Werke in 20 Bänden, Frankfurt/M.: Suhrkamp 1969-1971 (stw 601-620).

[30] Heisenberg, W. (1925). Über quantenmechanische Umdeutung kinematischer und mechanischer Beziehungen", Z. Phys. XXXIII, , 879ff.

[31] Heisenberg, W. (1977). Die Geschichte der Quantentheorie", in: Physik und Philosophie (Ullstein, Frankfurt etc), , 15-27.

[32] Helmholtz, H. (1847). Über die Erhaltung der Kraft (Reimer, Berlin) 
[33] Helmholtz, H. von (1911). Vorlesungen über die Dynamik discreter Massenpunkte (Barth, Leipzig, $2^{\text {nd }}$ ed.)

[34] Hund, F. Zur Deutung der Molekelspektren. I, Z. Phys. (1927). III. Bemerkungen über das Schwingungs- und Rotationsspektrum bei Molekeln mit mehr als zwei Kernen, 43 (1927) 805-826, 40(1927), 742-764.

[35] Leibniz, G. W. Specimen dynamicum pro admirandis naturae legibus circa corporum vires et mutuas actiones detegendis et ad suas causas revocandis, Acta erudit. Lipsiens. April ; Engl.: Essay in Dynamics showing the wonderful laws of nature concerning bodily forces and their interactions, and tracing them to their causes, http://www.earlymoderntexts.com/pdf/leibessa.pdftranslated, edited and commented by J. Bennett, June (2006).

[36] Messiah, A. (1999). Quantum Mechanics (Dover, New York), § I.15

[37] Newton, I. (1999). The Principia. Mathematical Principles of Natural Philosophy (A New Translation by I. Bernhard Cohen and Anne Whitman assisted by Julia Buden, Preceded by A Guide to Newton's Principia by I. Bernhard Cohen), Berkeley etc.: Univ. Calif. Press

[38] Nordheim, L. W. Zur Theorie der thermischen Emission und der Reflexion von Elektronen an Metallen, Z. Phys. (1928). , 46(1928), 833-855.

[39] Pauli, W. (1926). Quantentheorie", in: H. Geiger and K. Scheel (Eds.): Handbuch der Physik (Springer, Berlin), , 23, 1-278.

[40] Pauli, W. (1973). Wave Mechanics (Pauli Lectures of Physics, Ed. Ch. P. Enz (MIT Press, Cambridge, Mass., 1973), §35, 5

[41] Planck, M. (1900). Zur Theorie des Gesetzes der Energieverteilung im Normalspektrum", Verh. Dtsch. Phys. Ges., 2, , $237 \mathrm{ff}$.

[42] Schleich, W. P. (2001). Quantum Optics in Phase Space (Berlin etc., Wiley-VCH), , 282.

[43] Schrödinger, E. (1926). Quantisierung als Eigenwertproblem. Erste Mitteilung, Ann. Phys. [4] Zweite Mitteilung, 489-527; Dritte Mitteilung: Störungstheorie, mit Anwendung auf den Starkeffekt der Balmerlinien, 80 (1926) 437-490; Vierte Mitteilung, 81 (1926) 109-139; reprints in: Abhandlungen zur Wellenmechanik (Barth, Leipzig), 79(1926), 361-376.

[44] Schrödinger, E. (1933). The fundamental idea of wave mechanics" (Nobel award lecture, 1933)

[45] Suisky, D. (2009). Euler as Physicist, Berlin Heidelberg: Springer

[46] Suisky, D, \& Enders, P. (2001). Leibniz's Foundation of Mechanics and the Development of 18th Century Mechanics initiated by Euler", in: H. Poser (Ed.), Nihil sine ratione, Proc. VII Intern. Leibniz Congress, Berlin, http://www.leibniz-kongress.tu- 
berlin.de/webprogramm.html;http://www.information-philosophie.de/philosophie/ leibniz2001.html, 1247.

[47] Suisky, D, \& Enders, P. (2003). On the derivation and solution of the Schrödinger equation. Quantization as selection problem", Proc. $5^{\text {th }}$ Int. Symp. Frontiers of Fundamental Physics, Hyderabad (India), Jan. , 8-11.

[48] Weber, H. Über die Integration der partiellen Differentialgleichung..., Math. Ann. I ((1869).

[49] Weisstein, E. W. (2012). Fourier Transform", From MathWorld-A Wolfram Web Resource; http://mathworld.wolfram.com/FourierTransform.htmlMarch 13, 2012)

[50] Weizsäcker, C. F. v. (2002). Aufbau der Physik (dtv, München, $4^{\text {th }}$ ed.), 235.

[51] Weyl, H. (1950). The Theory of Groups in Quantum Mechanics (Dover, New York), § II.8

[52] Whittaker, E. T, \& Watson, G. N. A Course of Modern Analysis, Cambridge: Cambridge Univ. Press ${ }^{4} 1927$, new ed. (1996). (Cambr. Math. Libr. Ser.) 